\title{
Design of IIR Digital Differentiators Using Constrained Optimization
}

\author{
Rajeev C. Nongpiur, Member, IEEE, Dale J. Shpak, Senior Member, IEEE, and Andreas Antoniou, Life Fellow, IEEE
}

\begin{abstract}
A new optimization method for the design of fullband and lowpass IIR digital differentiators is proposed. In the new method, the passband phase-response error is minimized under the constraint that the maximum passband amplitude-response relative error is below a prescribed level. For lowpass IIR differentiators, an additional constraint is introduced to limit the average squared amplitude response in the stopband so as to minimize any high-frequency noise that may be present. Extensive experimental results are included, which show that the differentiators designed using the proposed method have much smaller maximum phase-response error for the same passband amplitude-response error and stopband constraints when compared with several differentiators designed using state-of-the-art competing methods.
\end{abstract}

Index Terms-Digital differentiators, IIR filter design, design of filters by optimization.

\section{INTRODUCTION}

D IGITAL differentiators are used in various fields of signal processing such as in the design of compensators in control systems [1], extracting information about transients in biomedical signal processing [2]-[4], analyzing signals in radar systems [5], and for edge detection in image processing [6]. Differentiators having perfectly linear phase response can be easily designed using FIR filters. However, in most applications perfectly linear phase response is not required and differentiators having approximately linear phase are quite acceptable. In such applications, IIR differentiators are more attractive than FIR differentiators for two main reasons: Firstly, they can satisfy the given filter specifications with a much lower filter order thereby reducing the computational requirement or the complexity of hardware in a hardware implementation and, secondly, they usually have a much smaller group delay thereby resulting in lower system delay.

The presence of the denominator polynomial in IIR filters renders their design more challenging than that of FIR filters because it results in highly nonlinear objective functions that require highly sophisticated optimization methods. As IIR filters lack the inherent stability of FIR filters, stability constraints must be incorporated in the design process to ensure that the

Manuscript received May 28, 2013; revised September 26, 2013 and December 17, 2013; accepted December 28, 2013. Date of publication January 27, 2014; date of current version March 10, 2014. The associate editor coordinating the review of this manuscript and approving it for publication was Prof. Jean-Christophe Pesquet. This work was supported by the Natural Sciences and Engineering Research Council of Canada.

The authors are with the Department of Electrical and Computer Engineering, University of Victoria, Victoria, BC V8W 2Y2, Canada (e-mail: rnongpiu@ece. uvic.ca; dshpak@ece.uvic.ca; aantoniou@ieee.org).

Digital Object Identifier 10.1109/TSP.2014.2302733 filter is stable, which means constraining the poles to lie within the unit circle of the $z$ plane.

Lowpass differentiators are appropriate when the signal of interest is at the low frequency end as they provide the advantage of reducing any high-frequency noise that may be present. In [7], [8], lowpass IIR differentiators have been designed by inverting the transfer function of lowpass integrators and then adjusting the denominator coefficients so that the poles lie within the unit circle. More recently in [9], two methods for designing lowpass IIR differentiators have been presented. In the first method, a fullband differentiator is cascaded with an appropriate lowpass filter while in the second method the numerator is realized as a linear-phase filter and the denominator is obtained using a constrained optimization method.

Earlier examples of fullband IIR differentiators are described in [10] although no method for their design is presented. In [11]-[15], fullband IIR differentiators are designed by taking the inverse of the transfer function of a fullband integrator and appropriately adjusting the denominator coefficients so that the poles lie within the unit circle. In [16], a sequential minimization procedure based on second-order transfer-function updates is used while in [17] an iterative quadratic programming approach with prescribed passband edge frequency is presented. The method in [17] uses a restrictive stability constraint that could affect the quality of the designs and, additionally, it requires that the group delay be specified. In [18] and [19], the differentiators are derived by taking an existing IIR differentiator and optimizing its pole-zero locations to improve the performance of the differentiator further.

In this paper, we propose a design method whereby the group-delay deviation with respect to the average group delay is minimized under the constraint that the maximum amplitude-response error be below a prescribed level. For lowpass IIR differentiators, we introduce an additional constraint to limit the average squared amplitude response in the stopband, so as to minimize any high-frequency noise that may be present. By representing the filter in polar form, a non-restrictive stability constraint characterized by a set of linear inequality constraints can be incorporated in the optimization algorithm. The group delay is included as an optimization variable to achieve improved design specifications. Procedures for designing fullband and lowpass IIR differentiators are then described. Experimental results show that differentiators designed using the proposed method have much smaller maximum phase-response error for the same passband error and stopband constraint than several known state-of-the-art methods.

The paper is organized as follows. In Section II, we formulate the problem as an iterative constrained optimization problem. In 
Section III, we describe procedures for designing fullband and lowpass IIR differentiators. In Section IV, performance comparisons between filters designed using the proposed method and known methods are carried out. Conclusion are drawn in Section V.

\section{The Optimization Problem}

In this section, we formulate the problem at hand as an iterative constrained optimization problem by approximating each update as a linear approximation step as was done in [20] and [21]. To this end, we derive formulas for the stability constraints, group-delay deviation, passband error, and stopband attenuation. Then, we incorporate the analytical results obtained within the framework of a constrained optimization problem.

A digital differentiator can be represented by the transfer function

$$
H(z)=H_{0} \frac{\prod_{m=1}^{J}\left(z-r_{a m}^{(1)} e^{j \theta_{a m}}\right)\left(z-r_{a m}^{(2)} e^{-j \theta_{a m}}\right)}{\prod_{m=1}^{J}\left(z-r_{b m}^{(1)} e^{j \theta_{b m}}\right)\left(z-r_{b m}^{(2)} e^{-j \theta_{b m}}\right)}
$$

where $J$ is the number of differentiator sections, $N=2 J$ is the differentiator order, and $H_{0}$ is a multiplier constant. An oddorder transfer function can be easily obtained by setting $r_{a 1}^{(1)}$ and $r_{b 1}^{(1)}$ to zero in the first section. [22]

The ideal response of a causal differentiator is of the form

$$
H_{d}(\omega)=j \omega e^{-j \tau \omega}, \quad 0<|\omega|<\pi
$$

where $\tau$ is the group delay. From (2), it is clear that at $\omega=0$ the amplitude response is zero while the phase characteristic has a discontinuity of $\pi$ and jumps between $-\pi / 2$ and $\pi / 2$ as frequency $\omega$ switches between $0_{-}$and $0_{+}$. Such a frequency response at $\omega=0$ can be realized by placing a zero at $z=1$ [10]. With this modification, the transfer function of the differentiator in (1) becomes

$$
\begin{array}{r}
H(\mathbf{c}, z)=H_{0}(z-1)\left(z-r_{a 1}\right) \\
\frac{\prod_{m=2}^{J}\left(z-r_{a m}^{(1)} e^{j \theta_{a m}}\right)\left(z-r_{a m}^{(2)} e^{-j \theta_{a m}}\right)}{\prod_{m=1}^{J}\left(z-r_{b m}^{(1)} e^{j \theta_{b m}}\right)\left(z-r_{b m}^{(2)} e^{-j \theta_{b m}}\right)}
\end{array}
$$

where

$$
\begin{gathered}
\mathbf{c}=\left[\begin{array}{lllllllllll}
r_{a 1} & r_{b 1}^{(1)} & r_{b 1}^{(2)} & \theta_{b 1} & r_{a 2}^{(1)} & r_{a 2}^{(2)} & \theta_{a 2} & r_{b 2}^{(1)} & r_{b 2}^{(2)} & \theta_{b 2} & \cdots \\
& & r_{a J}^{(1)} & r_{a J}^{(2)} & \theta_{a J} & r_{b J}^{(1)} & r_{b J}^{(2)} & \theta_{b J} & H_{0}
\end{array}\right]^{T}
\end{gathered}
$$

To ensure that the differentiator is stable, the poles of the transfer function must lie within the unit circle [22]. If $\epsilon_{s} \geq 0$ is a stability margin of the pole radius from unity, and $r_{b m}^{(1)}(k)$ and $r_{b m}^{(2)}(k)$ are the corresponding values of $r_{b m}^{(1)}$ and $r_{b m}^{(2)}$ at the start of the $k$ th iteration of the optimization, the stability conditions are given by

$$
\begin{array}{ll}
\mid r_{b m}^{(1)}(k)+\delta_{b m}^{(1)} \\
\mid \leq 1-\epsilon_{s} & \forall m \in[1, J] \\
r_{b m}^{(2)}(k)+\delta_{b m}^{(2)} \mid \leq 1-\epsilon_{s} & \forall m \in[1, J]
\end{array}
$$

where $\delta_{b m}^{(1)}$ and $\delta_{b m}^{(2)}$ are the corresponding updates for $r_{b m}^{(1)}(k)$ and $r_{b m}^{(2)}(k)$. Note that the conditions in (5) are convex inequality constraints and can, therefore, be incorporated within a convex optimization problem.

\section{A. Group Delay Deviation}

The group delay corresponding to the transfer function $H(\mathbf{c}, z)$ in (3) is given by

$$
\begin{aligned}
\tau_{h}(\mathbf{c}, \omega)= & \alpha(1,0, \omega)+\alpha\left(r_{a 1}, 0, \omega\right) \\
& +\sum_{m=2}^{J}\left[\alpha\left(r_{a m}^{(1)}, \theta_{a m}, \omega\right)+\alpha\left(r_{a m}^{(2)},-\theta_{a m}, \omega\right)\right] \\
& -\sum_{m=1}^{J}\left[\alpha\left(r_{b m}^{(1)}, \theta_{b m}, \omega\right)+\alpha\left(r_{b m}^{(2)},-\theta_{b m}, \omega\right)\right]
\end{aligned}
$$

where

$$
\alpha(r, \theta, \omega)= \begin{cases}-1 / 2 & r=1 \\ \frac{r \cos (\theta-\omega)-1}{r^{2}-2 r \cos (\theta-\omega)+1} & \text { otherwise }\end{cases}
$$

The group-delay deviation at frequency $\omega$ is given by

$$
e_{g}(\mathbf{x}, \omega)=\tau_{h}\left(\mathbf{c}, e^{j \omega}\right)-\tau
$$

where

$$
\mathbf{x}=\left[\mathbf{c}^{T} \tau\right]^{T}
$$

and $\tau$ is the desired group delay which may be an optimization variable. To incorporate the $L_{p}$ norm of the group-delay deviation, $\mathbf{E}_{p}^{(g d)}$, in an iterative optimization problem we can approximate $\mathbf{E}_{p}^{(g d)}$ for the $k$ th iteration by a linear approximation given by [20]

$$
\mathbf{E}_{p}^{(g d)}(k) \approx\left\|\mathbf{C}_{k} \boldsymbol{\delta}+\mathbf{d}_{k}\right\|_{p}
$$

where

$$
\begin{aligned}
& \mathbf{C}_{k}=\left[\begin{array}{c}
\kappa_{g} \nabla e_{g}\left(\mathbf{x}_{k}, e^{j \omega_{1}}\right)^{T} \\
\vdots \\
\kappa_{g} \nabla e_{g}\left(\mathbf{x}_{k}, e^{j \omega_{N_{p}}}\right)^{T}
\end{array}\right] \\
& \mathbf{d}_{k}=\left[\begin{array}{llll}
d_{1} & d_{2} & \cdots & d_{N_{p}}
\end{array}\right]^{T} \\
& d_{i}=\kappa_{g} e_{g}\left(\mathbf{x}_{k}, e^{j \omega_{i}}\right), \quad \omega_{i} \in \Psi_{p}
\end{aligned}
$$

$\mathbf{x}_{k}$ is the value of $\mathbf{x}$ in the $k$ th iteration, $\boldsymbol{\delta}$ is the update in $\mathbf{x}_{k}, \kappa_{g}$ is a constant, and $\Psi_{p}$ is the set of passband frequency sample 
points. The right-hand side of (10) is the $L_{p}$ norm of an affine function of $\delta$ and, therefore, it is convex with respect to $\delta$ [23].

\section{B. Passband Error}

If $H_{d}(\omega)$ is the desired frequency response of the differentiator in the passband and $\mathbf{c}_{k}$ is the value of vector $\mathbf{c}$ at the start of the $k$ th iteration, a passband error function at frequency $\omega$ can be defined as

$$
\begin{aligned}
e_{h}\left(\mathbf{c}_{k}, e^{j \omega}\right) & =W(\omega)\left[\left|H\left(\mathbf{c}_{k}, e^{j \omega}\right)\right|-\left|H_{d}(\omega)\right|\right] \\
& =W(\omega)\left[\left|H\left(\mathbf{c}_{k}, e^{j \omega}\right)\right|-|\omega|\right], \omega \in \Psi_{p}
\end{aligned}
$$

Constant absolute or relative error may be required and $W(\omega)$ can be chosen as unity or $1 /|\omega|$ depending upon the application. Note, however, that for constant absolute error, the relative error of the differentiator would tend to infinity as the frequency tends to zero; therefore, constant absolute error would not, typically, be of much practical interest and the design of differentiators with constant absolute error will not be considered further.

For the case of relative error, $e_{h}\left(\mathbf{c}_{k}, e^{j \omega}\right)$ can be expressed as

$$
e_{h}\left(\mathbf{c}_{k}, e^{j \omega}\right)=[P(\omega)-1], \omega \in \Psi_{p}
$$

where

$$
P(\omega)=\frac{\left|H\left(\mathbf{c}_{k}, e^{j \omega}\right)\right|}{|\omega|}
$$

Function $P(\omega)$ becomes indeterminate when $\omega=0$. To circumvent this problem, we set $z=e^{j \omega}$ and substitute (3) in (16) to obtain

$$
\begin{aligned}
P(\omega)= & \left|H_{0}\left[e^{j \omega}-r_{a 1}\right]\right| \\
& \cdot\left|\frac{-\omega \operatorname{sinc}^{2}(\omega / 2)+j 2 \operatorname{sinc} \omega}{2}\right| \\
& \cdot\left|\frac{\prod_{m=2}^{J}\left(e^{j \omega}-r_{a m}^{(1)} e^{j \theta_{a m}}\right)\left(e^{j \omega}-r_{a m}^{(2)} e^{-j \theta_{a m}}\right)}{\prod_{m=1}^{J}\left(e^{j \omega}-r_{b m}^{(1)} e^{j \theta_{b m}}\right)\left(e^{j \omega}-r_{b m}^{(2)} e^{-j \theta_{b m}}\right)}\right|
\end{aligned}
$$

where

$$
\operatorname{sinc}(x)= \begin{cases}\frac{1}{\sin x} & x=0 \\ x & \text { otherwise }\end{cases}
$$

Using the same approach as in Section II-A, the $L_{p}$ norm of the passband relative error, $e_{h}\left(\mathbf{c}_{k}, e^{j \omega}\right)$, in (15) can be expressed in matrix form as

$$
\mathbf{E}_{p}^{(p b)}(k) \approx\left\|\mathbf{D}_{k}^{(p b)} \boldsymbol{\delta}+\mathbf{f}_{k}^{(p b)}\right\|_{p}
$$

where

$$
\mathbf{D}_{k}^{(p b)}=\left[\begin{array}{cc}
\kappa_{p b} \nabla e_{h}\left(\mathbf{c}_{k}, e^{j \omega_{1}}\right)^{T} & 0 \\
\vdots & \vdots \\
\kappa_{p b} \nabla e_{h}\left(\mathbf{c}_{k}, e^{j \omega_{N_{p}}}\right)^{T} & 0
\end{array}\right], \quad \omega_{i} \in \Psi_{p}
$$

$$
\begin{aligned}
\mathbf{f}_{k}^{(p b)} & =\left[f_{1}^{(p b)} f_{2}^{(p b)} \cdots f_{N_{p}}^{(p b)}\right]^{T} \\
f_{i}^{(p b)} & =\kappa_{p b} e_{h}\left(\mathbf{c}_{k}, e^{j \omega_{i}}\right) \\
\boldsymbol{\delta} & =\left[\boldsymbol{\delta}_{c}^{T} \delta_{\tau}\right]^{T}
\end{aligned}
$$

Vector $\boldsymbol{\delta}_{c}$ in (23) is the update for $\mathbf{c}_{k}, \delta_{\tau}$ is a scalar update for $\tau$, and $\kappa_{p b}$ is a constant. The elements of the last column of $\mathbf{D}_{k}^{(p b)}$ in (20) are all zeros since (19) is independent of $\tau$.

\section{Stopband Amplitude Response}

The frequency response update for the differentiator at the $k$ th iteration is given by

$$
H\left(\mathbf{c}_{k}+\boldsymbol{\delta}_{c}, e^{j \omega}\right) \approx H\left(\mathbf{c}_{k}, e^{j \omega}\right)+\nabla H\left(\mathbf{c}_{k}, e^{j \omega}\right)^{T} \boldsymbol{\delta}_{c}
$$

The stopband noise that may be required to be attenuated may not always be white. If the spectrum of the stopband noise is known in advance, a weight $W_{s}(\omega)$ can be incorporated in (24) to emphasize frequency components with higher noise power as follows:

$$
\begin{aligned}
W_{s}(\omega) H\left(\mathbf{c}_{k}+\boldsymbol{\delta}_{c}, e^{j \omega}\right) \approx W_{s}(\omega)[ & H\left(\mathbf{c}_{k}, e^{j \omega}\right) \\
& \left.+\nabla H\left(\mathbf{c}_{k}, e^{j \omega}\right)^{T} \boldsymbol{\delta}_{c}\right]
\end{aligned}
$$

In such cases, $W_{s}(\omega)$ can correspond to the normalized magnitude spectrum of the noise in the stopband. If the stopband noise is white, as is assumed in all our experiments in Section IV, then $W_{s}(\omega)$ is set to unity.

By using the same approach as in Section II-B, the $L_{p}$ norm of the stopband weighted frequency response can be approximated as

$$
\mathbf{E}_{p}^{(s b)}(k) \approx\left\|\mathbf{D}_{k}^{(s b)} \boldsymbol{\delta}+\mathbf{f}_{k}^{(s b)}\right\|_{p}
$$

where

$$
\begin{aligned}
\mathbf{D}_{k}^{(s b)} & =\left[\begin{array}{cc}
\kappa_{s b} W_{s}\left(\omega_{1}\right) \nabla H\left(\mathbf{c}_{k}, e^{j \omega_{1}}\right)^{T} & 0 \\
\vdots & \vdots \\
\kappa_{s b} W_{s}\left(\omega_{N_{s}}\right) \nabla H\left(\mathbf{c}_{k}, e^{j \omega_{N_{s}}}\right)^{T} & 0
\end{array}\right], \quad \omega_{i} \in \Psi_{s} \\
\mathbf{f}_{k}^{(s b)} & =\left[\begin{array}{llll}
f_{1} & f_{2} & \cdots & f_{N_{s}}
\end{array}\right]^{T}, \quad \omega_{i} \in \Psi_{s} \\
f_{i} & =\kappa_{s b} W_{s}(\omega) H\left(\mathbf{c}_{k}, e^{j \omega_{i}}\right)
\end{aligned}
$$

$\Psi_{s}$ in (28) corresponds to the set of frequency points in the stopband and $\kappa_{s b}$ is a constant.

\section{Optimization Problem}

The optimization can be carried out by minimizing the groupdelay deviation under the constraints that the passband error and stopband attenuation are within prescribed levels. The design of a lowpass differentiator can be obtained by solving the optimization problem

$$
\text { minimize }\left\|e_{g}\left(\mathbf{x}, e^{j \omega}\right)\right\|_{p}
$$

subject to : passband error function $\leq \Gamma_{p b}$

stopband gain $\leq \Gamma_{s b}$

the differentiator is stable 
For the case of a fullband differentiator, the stopband constraint is not relevant and it is not included.

Using (5), (10), (19), and (26), the problem for the $k$ th iteration can be expressed as

$$
\begin{array}{rll}
\text { minimize } & \left\|\mathbf{C}_{k} \boldsymbol{\delta}+\mathbf{d}_{k}\right\|_{p} \\
\text { subject to : } & \left\|\mathbf{D}_{k}^{(p b)} \boldsymbol{\delta}+\mathbf{f}_{k}^{(p b)}\right\|_{p} \leq \Gamma_{p b} & \\
& \left\|\mathbf{D}_{k}^{(s b)} \boldsymbol{\delta}+\mathbf{f}_{k}^{(s b)}\right\|_{p} \leq \Gamma_{s b} & \\
& \|\boldsymbol{\delta}\|_{2} \leq \Gamma_{\text {small }} & \\
& \left|r_{b m}^{(1)}(k)+\delta_{b m}^{(1)}\right| \leq 1-\epsilon_{s} \quad \forall m \in[1, J] \\
& \left|r_{b m}^{(2)}(k)+\delta_{b m}^{(2)}\right| \leq 1-\epsilon_{s} \quad \forall m \in[1, J]
\end{array}
$$

where $\boldsymbol{\delta} \in \mathbf{R}^{6 J-1}$ is the optimization variable. The optimum value of $\delta$ is then used to update the optimizing parameters for the next iteration. Note that updates $\delta_{b m}^{(1)}$ and $\delta_{b m}^{(2)}$ are included in the update vector $\boldsymbol{\delta}$.

In the design of IIR differentiators, the typical approach is to minimize the maximum passband amplitude-response error and maximum phase-response error. For the former, this can be done by making the value of $p$ large when computing the $L_{p}$ norm for the parameter in (19). However, for the latter it is more appropriate to use the $L_{1}$ norm of (10) since the group delay is the negative of the derivative of the phase and minimization of the $L_{1}$ norm of the group-delay error tends to yield more reduction in the maximum phase-response error than the minimization of the $L_{\infty}$ norm. As in [20], we include a slack variable $\delta_{r l x}$ in the passband-error constraint in case the initialization filter does not satisfy the maximum passband-error constraint. With these modification, the problem in (31) becomes

$$
\begin{aligned}
& \text { minimize }\left\|\mathbf{C}_{k} \boldsymbol{\delta}+\mathbf{d}_{k}\right\|_{1}+V \delta_{r l x} \\
& \text { subject to : } \operatorname{sum}\left[\mathbf{C}_{k} \boldsymbol{\delta}+\mathbf{d}_{k}\right]=0 \\
& \left\|\mathbf{D}_{k}^{(p b)} \boldsymbol{\delta}+\mathbf{f}_{k}^{(p b)}\right\|_{\infty} \leq \Gamma_{p b}+\delta_{r l x} \\
& \left\|\mathbf{D}_{k}^{(s b)} \boldsymbol{\delta}+\mathbf{f}_{k}^{(s b)}\right\|_{2} \leq \Gamma_{s b} \\
& \|\boldsymbol{\delta}\|_{2} \leq \Gamma_{\text {small }}+\delta_{\text {rlx }} \\
& \delta_{r l x} \geq 0 \\
& \left|r_{b m}^{(1)}(k)+\delta_{b m}^{(1)}\right| \leq 1-\epsilon_{s} \quad \forall m \in[1, J] \\
& \left|r_{b m}^{(2)}(k)+\delta_{b m}^{(2)}\right| \leq 1-\epsilon_{s} \quad \forall m \in[1, J]
\end{aligned}
$$

where

$$
\operatorname{sum}[\mathbf{X}]=\sum_{i} x_{i}
$$

$\delta$ and $\delta_{r l x}$ are optimization variables and $V$ is a positive weighing factor for the relaxation variable.

Note that as in [20], the group delay can be fixed to a prescribed value or it can be optimized. In some applications it is desirable that the optimized group delay be small. In such cases, we can constrain the desired group delay $\tau$ in (9) to be below a prescribed upper bound $\Gamma_{g d}$. Such a constraint is given by

$$
\tau \leq \Gamma_{g d}+\delta_{r l x}
$$

where slack variable $\delta_{r l x}$ is included if $\tau$ is greater than $\Gamma_{g d}$ during initialization. The minimization of the group-delay deviation instead of the phase-response error in (32) would result in a sign ambiguity in the final solution. This can be corrected simply by checking the sign of the final solution and multiplying the transfer function by -1 if the sign is reversed.

The optimization problem in (32) can be easily expressed as a second-order cone programming (SOCP) problem as in [21] and solved using efficient SOCP solvers such as the one available in the SeDuMi optimization toolbox for MATLAB [24].

\section{Design of Digital DifFERENTIATORS}

In this section, we first describe a procedure for the design of the lowest even- and odd-order initialization IIR filters that would satisfy or nearly satisfy the passband amplitude-response constraint. We then use the transfer functions of these filters to construct initialization transfer functions for the design of differentiators. After that we describe a procedure for the design of digital differentiators and discuss a number of relevant practical issues.

\section{A. Design of Lowest-Order Initialization Filters}

The design of the lowest even- and odd-order initialization IIR filters that would satisfy or nearly satisfy the amplituderesponse constraint can be accomplished by using a modified version of the algorithm reported in [25] in which the absolute relative error is minimized instead of the squared amplituderesponse error.

If we let $z=e^{j \omega}$ in the transfer function of an IIR filter, namely,

$$
H_{m}(z)=\frac{\sum_{i=0}^{m} b_{i} z^{-i}}{\sum_{i=0}^{n} a_{i} z^{-i}}
$$

the squared amplitude response can be obtained as

$$
\begin{aligned}
\frac{N(\omega)}{D(\omega)}= & \left|H_{m}\left(e^{j \omega}\right)\right|^{2}=H_{m}\left(e^{j \omega}\right) H_{m}\left(e^{-j \omega)}\right. \\
= & \frac{p_{0}+\sum_{i=1}^{m} 2 p_{i} \cos (\omega i)}{q_{0}+\sum_{i=1}^{n} 2 q_{i} \cos (\omega i)}
\end{aligned}
$$

where $p_{-m}, \ldots, p_{m}$ and $q_{-n}, \ldots, q_{n}$ are the numerator and denominator coefficients, respectively, of the product $H_{m}(z) H_{m}\left(z^{-1}\right)$ such that $p_{i}=p_{-i}$ and $q_{i}=q_{-i}$. If $F_{d}(\omega)$ is the desired squared passband amplitude response of the differentiator, then the optimization algorithm in [25] can be used to obtain the filter coefficients that would satisfy the constraint

$$
\epsilon_{l}(\omega) \leq\left[\frac{N(\omega)}{D(\omega)}-F_{d}(\omega)\right] \leq \epsilon_{r}(\omega), \quad \forall \omega \in \Psi_{p}
$$


If $\delta_{r}$ is the maximum absolute relative error of the passband amplitude response, then

$$
\frac{1}{|\omega|}\left|\sqrt{\frac{N(\omega)}{D(\omega)}}-\sqrt{F_{d}(\omega)}\right| \leq \delta_{r}, \quad \forall \omega \in \Psi_{p}
$$

where $F_{d}(\omega)=\omega^{2}$. Now as shown in the Appendix, we can select $\epsilon_{l}(\omega)$ and $\epsilon_{r}(\omega)$ in (37) as

$$
\begin{aligned}
\epsilon_{r}(\omega) & =\left(2 \delta_{r}+\delta_{r}^{2}\right) \omega^{2} \\
\epsilon_{l}(\omega) & =\left(2 \delta_{r}-\delta_{r}^{2}\right) \omega^{2}
\end{aligned}
$$

For the design of a lowpass differentiator, an additional requirement is to limit the gain above the passband edge frequency so as to minimize any out-of-band high-frequency noise. One way to do this is to constrain the gain at $\omega=\pi$ to be below a certain threshold such that

$$
\frac{N(\pi)}{D(\pi)} \leq \Gamma_{p}^{2}
$$

where $\Gamma_{p}$ is the maximum allowable gain at $\omega=\pi$. Since the ideal gain of a fullband differentiator at $\omega=\pi$ is $\pi$, we can assume the upper limit for $\Gamma_{p}$ to be $\pi$. Consequently, the required IIR filters can be designed by solving the linear programming (LP) problem

$$
\begin{aligned}
\operatorname{minimize} & \nu \\
\text { subject to : } & N(\omega)-D(\omega)\left[\omega^{2}+\epsilon_{r}(\omega)\right]-\nu \leq 0 \\
& -N(\omega)+D(\omega)\left[\omega^{2}-\epsilon_{l}(\omega)\right]-\nu \leq 0 \\
& N(\omega) \geq 0 \\
& D(\omega) \geq 0+\rho_{s} \\
& N(\pi)-\Gamma_{p}^{2} D(\pi) \leq 0 \\
& \nu \geq 0
\end{aligned}
$$

where $\rho_{s}$ is a small positive constant used to ensure that the poles lie inside the unit circle. The above LP problem can be solved for $\omega \in \Psi_{p}$ with $\nu, p_{i}$, and $q_{i}$ as the optimization variables.

If the optimal value of $\nu$ is close to zero, that is, $\nu_{\mathrm{opt}} \leq e_{\mathrm{small}}$, then the solution would approximately satisfy the passband constraints and the next step is to recover the actual minimum-phase filter from the optimal values of $p_{i}$ and $q_{i}$. This is a straightforward step that can be carried out by using either spectral factorization [26] or a procedure described in [25].

For the design of a fullband differentiator, the lowest-order IIR filters that would satisfy the passband constraint can be designed by means of the following procedure:

Step 1: Initialize the passband error, $\delta_{r}$, and the passband sampling frequencies, $\Psi_{p}$, to the prescribed values. Also set the initial filter order, $M$, to 1 , and $\Gamma_{p}$ to a sufficiently large value greater than $\pi$.

Step 2: For filter order $M$, set $m=n=M$ in (36) and solve the LP problem in (42).

Step 3: If the optimal value of $\nu$ is close to zero $\left(\nu_{\mathrm{opt}} \leq\right.$ $\left.e_{\text {small }}\right)$, the passband specification is satisfied. Set $M_{\text {low }}=M$ and proceed to Step 4 . Otherwise, set $M=M+1$ and go to Step 2 .
Step 4: Use the optimal values of $p_{i}$ and $q_{i}$ to obtained the lowest-order filter and stop.

For the case of a lowpass differentiator, use steps 1 to 4 above and then continue with the following additional steps:

Step 5: Without changing the filter order, find the smallest value of $\Gamma_{p}$ between 0 and $\pi$ that would satisfy the passband constraint (i.e., $\nu_{\mathrm{opt}} \leq e_{\mathrm{small}}$ ) by solving the LP problem in (42) for different values of $\Gamma_{p}$ in the required range. This can be done by using a one-dimensional optimization procedure such as the golden-section search [23]. An accuracy of $10^{-2}$ is typically sufficient.

Step 6: Use the optimal values of $p_{i}$ and $q_{i}$ for the smallest value of $\Gamma_{p}$ to derive the lowest-order filter that would satisfy the passband error specification for the lowpass differentiator.

The next step is to design a second IIR filter of order $M_{\text {low }}-1$ that would have the smallest passband error, $\delta_{r}$, in the range $[0$, 1] and satisfy the constraint $\nu_{\mathrm{opt}} \leq e_{\mathrm{small}}$ while keeping $\Gamma_{p}$ larger than $\pi$. The first IIR filter would be of even or odd order. Consequently, the second IIR filter would be of odd or even order. It can be designed as in Step 5 in the above procedure. If $M_{\text {low }}=1$, which is the lowest possible order, the second IIR filter can be obtained by setting the filter order to 2 and then performing Steps 2 to 4 above for a fullband differentiator or Steps 2 to 6 for a lowpass differentiator.

The transfer functions of the two IIR filters obtained, $H_{\text {mag }}\left(z, M_{1}\right)$ and $H_{\text {mag }}\left(z, M_{2}\right)$, are given by

$$
H_{\text {mag }}(z, M)=\prod_{i=1}^{M} \frac{z-r_{a i} e^{j \theta_{a i}}}{z-r_{b i} e^{j \theta_{b i}}}
$$

where $M=M_{1}$ or $M_{2}$ and

$$
\begin{aligned}
& M_{1}=M_{\mathrm{low}} \\
& M_{2}= \begin{cases}2 & \text { if } M_{\mathrm{low}}=1 \\
M_{\mathrm{low}}-1 & \text { otherwise }\end{cases}
\end{aligned}
$$

\section{B. Initialization Filters for Differentiators}

To obtain initialization filters for differentiators of the desired filter orders, we add a number of biquadratic transfer functions to $H_{\text {mag }}\left(z, M_{1}\right)$ and $H_{\text {mag }}\left(z, M_{2}\right)$.

Two types of allpass transfer functions can be used. One possibility is to use

$$
H_{a p}^{(1)}\left(z, M_{a p}\right)= \begin{cases}1 & \text { if } M_{a p}=0 \\ \prod_{i=1}^{M_{a p}} G_{0} \frac{z-r_{i}^{-1} e^{j \theta_{i}}}{z-r_{i} e^{j \theta_{i}}} & \text { otherwise }\end{cases}
$$

where $M_{a p}$ is the order of the transfer function,

$$
\theta_{i}=\frac{(i-1) 2 \pi}{M_{a p}}
$$

and $G_{0}$ is a multiplier constant. The second possibility is to use $H_{a p}^{(2)}\left(z, M_{a p}\right)$ which can be obtained as follows: For an odd-order allpass transfer function, $H_{a p}^{(2)}\left(z, M_{a p}\right)$ is obtained by rotating the pole-zero positions of $H_{a p}^{(1)}\left(z, M_{a p}\right)$ by $\pi$ radians in the $z$ plane; on the other hand, for an even-order allpass transfer 
function, $H_{a p}^{(2)}\left(z, M_{a p}\right)$ is obtained by rotating $H_{a p}^{(1)}\left(z, M_{a p}\right)$ by $\pi / 2$ radians either in the clockwise or counter-clockwise direction. Note that if the order of the allpass transfer function is a multiple of 4 , it can be easily shown that $H_{a p}^{(1)}\left(z, M_{a p}\right)$ and $H_{a p}^{(2)}\left(z, M_{a p}\right)$ are identical.

On the basis of the above principles, four initialization transfer functions are possible for differentiators given by

$$
\begin{aligned}
& H_{\text {init } 1}(z)=H_{\mathrm{mag}}\left(z, M_{1}\right) \cdot H_{a p}^{(1)}\left(z, M_{d}-M_{1}\right) \\
& H_{\mathrm{init} 2}(z)=H_{\mathrm{mag}}\left(z, M_{1}\right) \cdot H_{a p}^{(2)}\left(z, M_{d}-M_{1}\right) \\
& H_{\mathrm{init} 3}(z)=H_{\mathrm{mag}}\left(z, M_{2}\right) \cdot H_{a p}^{(1)}\left(z, M_{d}-M_{2}\right) \\
& H_{\mathrm{init} 4}(z)=H_{\text {mag }}\left(z, M_{2}\right) \cdot H_{a p}^{(2)}\left(z, M_{d}-M_{2}\right)
\end{aligned}
$$

where $M_{d}$ is the differentiator order. Note that $H_{\text {init } 1}(z)$ and $H_{\text {init } 2}(z)$ are valid only if $M_{d} \geq M_{1}$ while $H_{\text {init3 }}(z)$ and $H_{\text {init } 4}(z)$ are valid only if $M_{d} \geq M_{2}$.

The above four distinct initialization transfer functions often lead to different solutions and a good strategy would be to design a differentiator with each one of them and then select the best solution for the application at hand.

\section{Passband Phase-Response Error for Differentiators}

If the average passband group delay of the differentiator is given by

$$
\bar{\tau}=\frac{1}{\omega_{p}} \int_{0}^{\omega_{p}} \tau_{h}(\omega) d \omega
$$

where $\omega_{p}$ is the passband edge frequency and $\tau_{h}(\omega)$ is the group delay, then the ideal phase response of the differentiator is given by

$$
\phi_{\text {ideal }}(\omega)=\frac{\pi}{2}-\omega \bar{\tau}, \quad \omega \in[0,2 \pi]
$$

The phase-response error can be obtained as

$$
e_{\phi}(\omega)=\phi_{h}(\omega)-\phi_{\text {ideal }}(\omega), \quad e_{\phi}(\omega) \in[-\pi, \pi]
$$

where $\phi_{h}(\omega)$ is the actual phase response of the differentiator. Consequently, the maximum peak-to-peak phase-response error in degrees is given by

$$
\xi_{\phi}=\frac{180}{\pi}\left[\sup _{\omega} e_{\phi}(\omega)-\inf _{\omega} e_{\phi}(\omega)\right]
$$

Parameter $\xi_{\phi}$ will be referred to as the maximum phase-response error hereafter.

\section{Design Procedure for Differentiators}

The design of digital differentiators that would satisfy prescribed specifications can be carried out by using the following procedure:

Step 1: Compute the two lowest-order transfer functions, $H_{\text {mag }}\left(z, M_{1}\right)$ and $H_{\text {mag }}\left(z, M_{2}\right)$, using the procedure in Section III-A.

Step 2: Set the desired differentiator order to $M_{d}$ and compute the initialization filters in (48).
Step 3: Solve the optimization problem in (32) for all the initialization filters derived in Step 2. For the fullband differentiator set $\Gamma_{s b}$ to a large value, say, 10000 , while for the lowpass differentiator set it to the prescribed value.

Step 4: Select the solution that has the smallest maximum phase-response error $\xi_{\phi}$ and at the same time satisfies the passband error constraint; for the lowpass differentiator, the solution should also satisfy the stopband constraint.

Step 5: If a solution is found that satisfies the phase-error specification in Step 4, stop. Otherwise, set $M_{d}=$ $M_{d}+1$ and go to Step 2 .

\section{E. Special Case for Differentiators With Fixed Group Delay}

In general, the average group delay of fullband differentiators with optimized group delay increases as the order of the differentiator is increased. The value of the average group delay usually follows that of the ideal fullband causal differentiator where the group delay is confined to $\tau_{n}$ samples where $\tau_{n}$ is defined as

$$
\tau_{n}=0.5+n, \quad n \text { is a nonnegative integer }
$$

In applications where the order of the differentiator is large, it may be desirable to have a differentiator with a smaller group delay at the expense of increased in amplitude- and/or phaseresponse error. In such applications, a modified version of the design method in Section III-A can be used. Rather than finding the lowest-order filter that would satisfy the amplitude-response constraints, for differentiators with the smallest possible group delay we start from the opposite end by finding the prescribed highest and second-highest order filters that would satisfy the amplitude-response constraints and then using the procedure in Section III-B we obtain the initialization filters. In this way, as the desired group delay is increased, the order of the filter that would satisfy the amplitude-response constraint is progressively decreased.

The same approach can be used for lowpass differentiators with fixed group delay.

\section{F. Practical Considerations}

The frequency-dependent parameters are evaluated at frequency points that are sampled between $-\pi$ and $\pi$, such that the sample points between $-\pi$ and 0 are the negatives of the sample points between 0 and $\pi$. To reduce the number of sample points and at the same time prevent spikes in the passband amplitude-response error function, the nonuniform variable sampling technique described in Chapter 16 of [22] can be used. Unlike the passband amplitude-response error, which is an $L_{\infty}$ norm, the group delay and stopband errors are $L_{1}$ and $L_{2}$ norms, respectively, and hence the technique in [22] is not applicable. Therefore, a uniform sampling is used for these error functions.

The weight factor $V$ for the relaxation parameter $\delta_{\mathrm{rlx}}$ in (32) should not be too small, say, smaller than 100 , as this could make the optimization algorithm unstable and prevent it from converging; at the same time, it should not be too large, say, larger 
than 10000 , as this can slow down the convergence. Values of $V$ in the range 500 to 5000 were found to give good results.

To ensure that the optimization is not prematurely terminated, the optimization algorithm is stopped if the relative reduction in the $Q$ value is less that the prescribed maximum value typically over the 40 most recent iterations, as was done in [20].

\section{EXPERIMENTAL RESULTS}

In this section, we provide comparative experimental results to demonstrate the efficiency of the proposed method. Twelve design examples of various differentiator types are considered. Parameters $\Gamma_{\text {small }}$ and $V$ in (32) were set to 0.01 and 1000 , respectively. The allpass transfer function, $H_{a p}^{(1)}\left(z, M_{a p}\right)$, in (46) was initialized with $r_{k}=0.9$. The default maximum pole radius was set to 0.98 . A normalized sampling frequency of $2 \pi$ was assumed in all design examples. The number of virtual and actual sample frequencies used in the nonuniform sampling technique [22] over the frequency range $-\omega_{p}$ to $\omega_{p}$ were 2000 and 68 , respectively. Eight of the actual sample frequencies were uniformly distributed near the passband edge with a separation of $7.8 \times 10^{-4} \mathrm{rad} / \mathrm{s}$ between them. The group delay and stopband parameters, on the other hand, were sampled and evaluated using 800 uniformly sampled frequencies in the interval $[-\pi, \pi]$.

The stopband noise for the lowpass differentiators was assumed to be white Guassian and its power was proportional to the average squared stopband amplitude response and is given by

$$
P_{s b}=\frac{1}{\pi-\omega_{p}} \int_{\omega_{p}}^{\pi}\left|H\left(e^{j \omega}\right)\right|^{2} d \omega
$$

In Sections IV-A to IV-D below, we compare the proposed design method with a number of state-of-the-art methods for the design of digital differentiators including the methods in [9], [10], [12], [13], [15], and [16].

\section{A. Examples 1, 2, and 3}

The competing differentiators for Examples 1, 2, and 3 correspond to the third example in [10], the second example in [12], and the second example in [13], respectively. The required design specifications for these differentiators are given in Tables I, III, and V and the results obtained are summarized in Tables II, IV, and VI. The relative amplitude- and phase-response errors for Example 1 are plotted in Fig. 1. As can be seen in Fig. 1 and Tables II, IV, and VI, the IIR differentiators designed using the proposed method have much smaller maximum phase-response error for practically the same relative error in the amplitude response as the designs obtained with the competing methods. The differentiators reported in [15], [16] have poor amplitude and phase responses close to the zero frequency due to the absence of a zero at point $(1,0)$ of the $z$ plane, while the one in [14] has a phase-response error that is very large throughout the passband. For these reasons, we relegate the comparison of our differentiators with those in [15], [16], and [14] to the weblink document mentioned in [27].
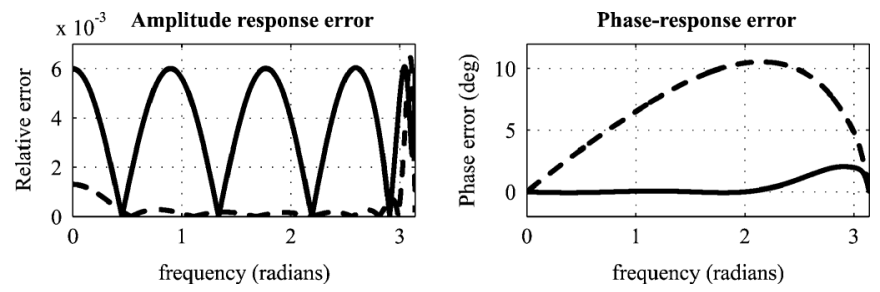

Fig. 1. Plots of relative amplitude-response error and phase-response error for proposed method 2 (solid curves) and the method in [10] (dashed curves) for Example 2.

TABLE I

FULLBAND DIFFERENTIATOR SPECIFICATIONS FOR EXAMPLE 1

\begin{tabular}{||l|c||}
\hline \hline Parameters & Values \\
\hline Maximum rel. error, $\delta_{r}$ & 0.0065 \\
Maximum pole radius & 0.98 \\
\hline \hline
\end{tabular}

TABLE II

DESIGN RESUlts FOR EXAMPLE 1 (FULLbAND DiFFERENTIATOR)

\begin{tabular}{||l|c|c|c||}
\hline \hline Parameters & $\begin{array}{c}\text { Proposed } \\
\text { method 1 }\end{array}$ & $\begin{array}{c}\text { Proposed } \\
\text { method 2 }\end{array}$ & $\begin{array}{c}\text { Method } \\
\text { in [10] }\end{array}$ \\
\hline Filter order & 4 & 6 & 6 \\
Max. rel. error, $\delta_{r}$ & 0.006 & 0.0061 & 0.0065 \\
Avg. group delay, $\bar{\tau}$ & 2.5 & 3.5 & 0.5 \\
Max. phase error, $\xi_{\phi}$ & 3.72 & 2.12 & 10.53 \\
\hline \hline
\end{tabular}

TABLE III

FULLBAND DIFFERENTIATOR SPECIFICATIONS FOR EXAMPLE 2

\begin{tabular}{||l|c||}
\hline \hline Parameters & Values \\
\hline Maximum rel. error, $\delta_{r}$ & 0.055 \\
Maximum pole radius & 0.98 \\
\hline \hline
\end{tabular}

TABLE IV

DESIGN Results For EXAMPLE 2 (FULlBAND DifFERENTIATOR)

\begin{tabular}{||l|c|c|c||}
\hline \hline Parameters & $\begin{array}{c}\text { Proposed } \\
\text { method 1 }\end{array}$ & $\begin{array}{c}\text { Proposed } \\
\text { method 2 }\end{array}$ & $\begin{array}{c}\text { Method } \\
\text { in [12] }\end{array}$ \\
\hline Filter order & 2 & 3 & 3 \\
Max. rel. error, $\delta_{r}$ & 0.05 & 0.05 & 0.055 \\
Avg. group delay, $\bar{\tau}$ & 0.5 & 1.5 & 0.5 \\
Max. phase error, $\xi_{\phi}$ & 7.12 & 2.06 & 12.05 \\
\hline \hline
\end{tabular}

TABLE V

FULLBAND DiFFERENTIATOR SPECIFICATIONS FOR EXAMPLE 3

\begin{tabular}{||l|c||}
\hline \hline Parameters & Values \\
\hline Maximum rel. error, $\delta_{r}$ & 0.035 \\
Maximum pole radius & 0.98 \\
\hline \hline
\end{tabular}

\section{B. Examples 4, 5, 6, and 7}

The design specifications for Examples 4 to 7 are given in Tables VII, IX, XI, and XIII, respectively. The competing differentiators for each of the examples correspond to the fourth, sixth, eighth, and thirteenth examples in [9], respectively. Tables VIII, X, XII and XIV and Fig. 2 show that the IIR differentiators designed using the proposed method have much smaller maximum phase-response error for practically the same passband relative amplitude-response error and average squared-amplitude response in the stopband as the designs obtained with the competing method in [9]. 
TABLE VI

DESIGN RESUlTS FOR EXAMPLE 3 (FULLBAND DIFFERENTIATOR)

\begin{tabular}{||l|c|c|c||}
\hline \hline Parameters & $\begin{array}{c}\text { Proposed } \\
\text { method 1 }\end{array}$ & $\begin{array}{c}\text { Proposed } \\
\text { method 2 }\end{array}$ & $\begin{array}{c}\text { Method } \\
\text { in [13] }\end{array}$ \\
\hline Filter order & 2 & 3 & 3 \\
Max. rel. error, $\delta_{r}$ & 0.031 & 0.031 & 0.0317 \\
Avg. group delay, $\bar{\tau}$ & 0.5 & 1.5 & 0.5 \\
Max. phase error, $\xi_{\phi}$ & 8.26 & 3.26 & 12.05 \\
\hline \hline
\end{tabular}

TABLE VII

LOWPASS DIFFERENTIATOR SPECIFICATIONS FOR EXAMPLE 4

\begin{tabular}{||l|c||}
\hline \hline Parameters & Values \\
\hline Maximum rel. error, $\delta_{r}$ & 0.015 \\
Maximum ASAR in SB & 1.2 \\
Passband edge, rad/s & $0.7 \pi$ \\
Maximum pole radius & 0.98 \\
\hline \hline
\end{tabular}

ASAR: average squared amplitude-response; SB: stopband
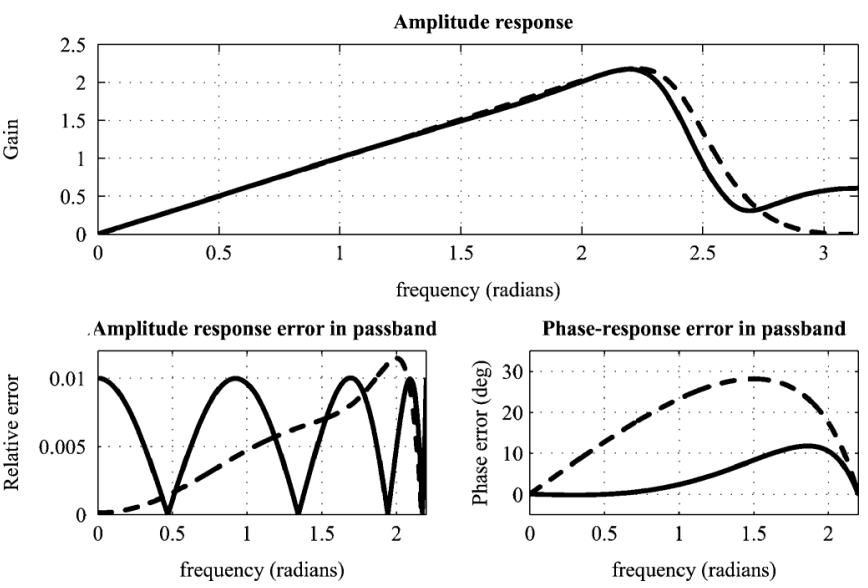

Fig. 2. Plots of amplitude response, relative amplitude-response error and phase-response error for the proposed method (solid curves) and the method in [9] (dashed curves) for Example 4.

TABLE VIII

DESIGN RESULTS FOR EXAMPLE 4 (LOWPASS DiFFERENTIATOR)

\begin{tabular}{||l|c|c||}
\hline \hline Parameters & $\begin{array}{c}\text { Proposed } \\
\text { method }\end{array}$ & $\begin{array}{c}\text { Method } \\
\text { in [9] }\end{array}$ \\
\hline Filter order & 4 & 4 \\
Max. rel. error, $\delta_{r}$ & 0.01 & 0.0115 \\
ASAR in SB, $P_{s b}$ & 1.09 & 1.184 \\
Avg. group delay in PB, $\bar{\tau}$ & 2.02 & 1.24 \\
Max. phase error, $\xi_{\phi}$ & 12 & 28.16 \\
\hline
\end{tabular}

ASAR: average squared amplitude-response; SB: stopband; PB: passband

TABLE IX

LOWPASS DiFFERENTIATOR SPECIFICATIONS FOR EXAMPLE 5

\begin{tabular}{||l|c||}
\hline \hline Parameters & Values \\
\hline Maximum rel. error, $\delta_{r}$ & 0.016 \\
Maximum ASAR in SB & 0.45 \\
Passband edge, rad/s & $0.29 \pi$ \\
Maximum pole radius & 0.98 \\
\hline
\end{tabular}

ASAR: average squared amplitude-response; SB: stopband

\section{Examples 8, 9, and 10}

In Example 8, we have designed fullband differentiators with fixed and optimized group delays using the proposed method and compared our designs with a competing differentiator taken from ([19], (21)). The design specifications are
TABLE $X$

DESIGN RESUlTS FOR EXAMPLE 5 (LOWPASS DIFFERENTIATOR)

\begin{tabular}{||l|c|c|c||}
\hline \hline Parameters & $\begin{array}{c}\text { Proposed } \\
\text { method 1 }\end{array}$ & $\begin{array}{c}\text { Proposed } \\
\text { method 2 }\end{array}$ & $\begin{array}{c}\text { Method } \\
\text { in [9] }\end{array}$ \\
\hline Filter order & 4 & 5 & 5 \\
Max. rel. error, $\delta_{r}$ & 0.015 & 0.015 & 0.0155 \\
ASAR in SB, $P_{s b}$ & 0.397 & 0.397 & 0.418 \\
Avg. group delay in PB, $\bar{\tau}$ & 3.71 & 7.15 & 2.53 \\
Max. phase error, $\xi_{\phi}$ & 1.52 & 0.30 & 8.26 \\
\hline
\end{tabular}

ASAR: average squared amplitude-response; SB: stopband; PB: passband

TABLE XI

LOWPASS DIFFERENTIATOR SPECIFICATIONS FOR EXAMPLE 6

\begin{tabular}{||l|c||}
\hline \hline Parameters & Values \\
\hline Maximum rel. error, $\delta_{r}$ & 0.04 \\
Maximum ASAR in SB & 0.55 \\
Passband edge, rad/s & $0.3 \pi$ \\
Maximum pole radius & 0.98 \\
\hline
\end{tabular}

ASAR: average squared amplitude-response; SB: stopband

TABLE XII

DESIGN RESUlts FOR EXAMPLE 6 (LOWPASS DIFFERENTIATOR)

\begin{tabular}{||l|c|c|c||}
\hline \hline Parameters & $\begin{array}{c}\text { Proposed } \\
\text { method 1 }\end{array}$ & $\begin{array}{c}\text { Proposed } \\
\text { method 2 }\end{array}$ & $\begin{array}{c}\text { Method } \\
\text { in [9] }\end{array}$ \\
\hline Filter order & 3 & 4 & 4 \\
Max. rel. error, $\delta_{r}$ & 0.035 & 0.035 & 0.036 \\
ASAR in SB, $P_{s b}$ & 0.498 & 0.494 & 0.503 \\
Avg. group delay in PB, $\bar{\tau}$ & 3.37 & 3.37 & 2.08 \\
Max. phase error, $\xi_{\phi}$ & 1.71 & 0.0032 & 5.25 \\
\hline
\end{tabular}

ASAR: average squared amplitude-response; SB: stopband; PB: passband

TABLE XIII

LOWPASS DIFFERENTIATOR SPECIFICATIONS FOR EXAMPLE 7

\begin{tabular}{||l|c||}
\hline \hline Parameters & Values \\
\hline Maximum rel. error, $\delta_{r}$ & 0.07 \\
Maximum ASAR in SB & 0.95 \\
Passband edge, rad/s & $0.5 \pi$ \\
Maximum pole radius & 0.98 \\
\hline
\end{tabular}

ASAR: average squared amplitude-response; SB: stopband

TABLE XIV

DESIGN RESUlTS FOR EXAMPLE 7 (LOWPASS DIFFERENTIATOR)

\begin{tabular}{||l|c|c|c||}
\hline \hline Parameters & $\begin{array}{c}\text { Proposed } \\
\text { method 1 }\end{array}$ & $\begin{array}{c}\text { Proposed } \\
\text { method 2 }\end{array}$ & $\begin{array}{c}\text { Method } \\
\text { in [9] }\end{array}$ \\
\hline Filter order & 3 & 5 & 5 \\
Max. rel. error, $\delta_{r}$ & 0.06 & 0.06 & 0.067 \\
ASAR in SB, $P_{s b}$ & 0.939 & 0.939 & 0.944 \\
Avg. group delay in PB, $\bar{\tau}$ & 2.31 & 4.46 & 1.66 \\
Max. phase error, $\xi_{\phi}$ & 1.74 & 0.025 & 11.75 \\
\hline
\end{tabular}

ASAR: average squared amplitude-response; SB: stopband; PB: passband

given in Table XV and the results obtained are summarized in Table XVI. From these results, we observe that differentiators designed with the proposed method have much smaller maximum phase-response error for practically the same passband relative amplitude-response error and average squared-amplitude response in the stopband. Note that the differentiator with optimized group delay has smaller phase-response error than the differentiator with fixed group delay but larger average group delay.

In Examples 9 and 10, we compare lowpass differentiators where the group delay of the differentiator in the proposed 
TABLE XV

FULLBAND DifFERENTIATOR SPECIFICATIONS FOR EXAMPLE 8

\begin{tabular}{||l|c||}
\hline \hline Parameters & Values \\
\hline Maximum rel. error, $\delta_{r}$ & 0.15 \\
Maximum pole radius & 0.98 \\
\hline \hline
\end{tabular}

TABLE XVI

DESIGN RESUlts FOR EXAMPLE 8 (FULLBAND DifFERENTIATOR)

\begin{tabular}{||l|c|c|c||}
\hline \hline Parameters & $\begin{array}{c}\text { Proposed } \\
\text { method 1 } \\
\text { (OGD) }\end{array}$ & $\begin{array}{c}\text { Proposed } \\
\text { method 2 } \\
\text { (FGD) }\end{array}$ & $\begin{array}{c}\text { Method } \\
\text { in [19] }\end{array}$ \\
\hline Filter order & 4 & 4 & 4 \\
Max. rel. error, $\delta_{r}$ & 0.1 & 0.1 & 0.11 \\
Avg. group delay, $\bar{\tau}$ & 3.5 & 0.5 & 0.5 \\
Max. phase error, $\xi_{\phi}$ & 0.00056 & 7.44 & 11.27 \\
\hline
\end{tabular}

OGD: optimized group delay; FGD: fixed group delay

TABLE XVII

DESIGN RESULTS FOR EXAMPLE 9 (LOWPASS DIFFERENTIATOR)

\begin{tabular}{||l|c|c||}
\hline \hline Parameters & $\begin{array}{c}\text { Proposed } \\
\text { method } \\
\text { (CGD) }\end{array}$ & $\begin{array}{c}\text { Method } \\
\text { in [9] }\end{array}$ \\
\hline Filter order & 5 & 5 \\
Max. rel. error, $\delta_{r}$ & 0.0143 & 0.0155 \\
ASAR in SB, $P_{s b}$ & 0.396 & 0.418 \\
Avg. group delay in PB, $\bar{\tau}$ & 1.76 & 2.53 \\
Max. phase error, $\xi_{\phi}$ & 5.66 & 8.26 \\
\hline
\end{tabular}

ASAR: average squared amplitude-response; SB: stopband;

PB: passband; CGD: constrained group delay

TABLE XVIII

DESIGN RESUlTS FOR EXAMPLE 10 (LOWPASS DifFERENTIATOR)

\begin{tabular}{||l|c|c||}
\hline \hline Parameters & $\begin{array}{c}\text { Proposed } \\
\text { method } \\
\text { (CGD) }\end{array}$ & $\begin{array}{c}\text { Method } \\
\text { in [9] }\end{array}$ \\
\hline Filter order & 4 & 4 \\
Max. rel. error, $\delta_{r}$ & 0.035 & 0.036 \\
ASAR in SB, $P_{s b}$ & 0.498 & 0.503 \\
Avg. group delay in PB, $\bar{\tau}$ & 1.452 & 2.08 \\
Max. phase error, $\xi_{\phi}$ & 2.63 & 5.25 \\
\hline
\end{tabular}

ASAR: average squared amplitude-response; SB: stopband;

PB: passband; CGD: constrained group delay

method is constrained to be equal to or less than that in the competing design; this is done by incorporating the inequality constraint in (34) in the optimization problem in (32). To observe how the performance changes with and without the group-delay constraint, we have used the design specifications and competing differentiators in Examples 7 and 8 for Examples 9 and 10, respectively. The design results for these two examples are tabulated in Tables XVII and XVIII. The poles and zeros for our proposed designs are given in [27]. From the results, we observe that the proposed design method yields differentiators that have smaller phase-response errors and average group delay than the competing methods. Upon comparing the designs in Examples 9 and 10 obtained with our method with the corresponding designs in Examples 7 and 8 obtained with our method, we observe that the designs in Examples 9 and 10 offer lower group delay at the expense of increased phase-response error.
TABLE XIX

FULLBAND DIFFERENTIATOR SPECIFICATIONS FOR EXAMPLE 11

\begin{tabular}{||l|c||}
\hline \hline Parameters & Values \\
\hline Maximum rel. error, $\delta_{r}$ & 0.005 \\
Maximum pole radius & 0.98 \\
\hline \hline
\end{tabular}

TABLE XX

EXAMPLE 11: COMPARISON BETWEEN THE IIR DIFFERENTIATORS AND AN EQUIVALENT FIR DIFFERENTIATOR (FULLBAND DIFFERENTIATOR)

\begin{tabular}{||l|c|c|c|c||}
\hline \hline Parameters & $\begin{array}{c}\text { IIR } \\
\text { Diff 1 }\end{array}$ & $\begin{array}{c}\text { IIR } \\
\text { Diff 2 }\end{array}$ & $\begin{array}{c}\text { IIR } \\
\text { Diff 3 }\end{array}$ & $\begin{array}{c}\text { FIR } \\
\text { Diff }\end{array}$ \\
\hline Filter order & 3 & 4 & 5 & 41 \\
Max. rel. error, $\delta_{r}$ & 0.0046 & 0.0046 & 0.0046 & 0.0047 \\
Avg. group delay, $\bar{\tau}$ & 0.5 & 1.5 & 3.5 & 20.5 \\
Max. phase error, $\xi_{\phi}$ & 10.20 & 5.58 & 2.77 & 0 \\
No. of multiplications & 7 & 9 & 11 & 21 \\
No. of additions & 6 & 8 & 10 & 41 \\
No of delays & 3 & 4 & 5 & 41 \\
\hline \hline
\end{tabular}

TABLE XXI

LOWPASS DIFFERENTIATOR SPECIFICATIONS FOR EXAMPLE 12

\begin{tabular}{||l|c||}
\hline \hline Parameters & Values \\
\hline Maximum rel. error, $\delta_{r}$ & 0.0095 \\
Maximum ASAR in SB & 0.65 \\
Maximum pole radius & 0.98 \\
\hline
\end{tabular}

ASAR: average squared amplitude-response; SB: stopband

TABLE XXII

EXAMPLE 12: COMPARISON BETWEEN THE LOWPASS IIR DIFFERENTIATORS AND AN EQUIVALENT FIR DIFFERENTIATOR (LOWPASS DIFFERENTIATOR)

\begin{tabular}{||l|c|c|c|c||}
\hline \hline Parameters & $\begin{array}{c}\text { IIR } \\
\text { Diff 1 }\end{array}$ & $\begin{array}{c}\text { IIR } \\
\text { Diff 2 }\end{array}$ & $\begin{array}{c}\text { IIR } \\
\text { Diff 3 }\end{array}$ & $\begin{array}{c}\text { FIR } \\
\text { Diff }\end{array}$ \\
\hline Filter order & 4 & 5 & 6 & 60 \\
Max. rel. error, $\delta_{r}$ & 0.009 & 0.009 & 0.009 & 0.0091 \\
ASAR in SB, $P_{s b}$ & 0.597 & 0.597 & 0.597 & 0.606 \\
Avg. group delay in PB, $\bar{\tau}$ & 1.31 & 4.11 & 4.39 & 30 \\
Max. phase error, $\xi_{\phi}$ & 8.91 & 2.41 & 0.13 & 0 \\
No. of multiplications & 9 & 11 & 13 & 31 \\
No. of additions & 8 & 10 & 12 & 60 \\
No of delays & 4 & 5 & 6 & 60 \\
\hline \hline
\end{tabular}

ASAR: average squared amplitude-response; SB: stopband; PB: passband

\section{Examples 11 and 12}

In Examples 11 and 12, we compare fullband and lowpass IIR differentiator designs with a corresponding optimal FIR design. The design specifications for these examples are given in Tables XIX and XXI, respectively. The optimization was carried out for various differentiator orders by varying the number of additional first-order filter sections. A differentiator order of $N=41$ was required to satisfy the specifications in Table XIX for the fullband FIR differentiator and this was designed using the Remez Exchange algorithm described in Chapter 15 of [22]. On the other hand, the specification in Table XXI for the lowpass FIR differentiator required an order of $N=60$ and the number of zeros at point $[-1,0]$ in the $z$ plane was set to $K=27$. This was designed using the Selesnick-Type III design method [28]. The results obtained and the number of arithmetic operations per sampling period are presented in Tables XX and XXII. We have assumed a cascade realization of second-order sections both for the IIR and FIR differentiators. For the IIR differentiators, we have assumed a direct-canonic realization which would require a total of $2 N+1$ multiplications, $2 N$ additions, and $N$ unit delays per sampling period where $N$ is the differentiator 
order [22]. In the case of the FIR differentiator, $(N+1) / 2$ multiplications, $N$ additions, and $N$ unit delays would be required per sampling period in view of the symmetry property of the transfer function coefficients in constant group-delay filters. From Tables XX and XXII, we observe a clear trade-off between filter complexity and group delay versus maximum phase-response error. It is apparent that the IIR differentiators offer a significant reduction in the number of arithmetic operations and system latency but at the cost of a nonzero phase-response error. For most applications, a perfectly linear-phase response is not required and a value of $\xi_{\phi}$ in the range of 1 to 10 , depending on the application, would be entirely acceptable. In such applications, a significantly more economical and efficient IIR design would be possible.

\section{E. Examples 13 to 18}

Additional comparisons of IIR fullband differentiators to demonstrate the effectiveness of our proposed method are included in the online document in [27]. The competing differentiators also include design examples taken from [11], [14], and [18].

\section{CONCLUSION}

A method for the design of fullband and lowpass IIR digital differentiators that would satisfy prescribed specifications has been described. The passband phase-response error is minimized under the constraint that the maximum relative amplitude-response error is below a prescribed level. For lowpass IIR differentiators, an additional constraint is introduced to limit the stopband average squared amplitude response so as to minimize any high-frequency noise that may be present.

The experimental results presented show that the differentiators designed using the proposed method have much smaller maximum phase-response error for the same passband relative amplitude-response error and stopband constraints when compared with differentiators designed with several state-of-the-art competing methods. Our results also show that nearly linear-phase IIR differentiators can offer some important advantages over their perfectly linear-phase FIR counterparts such as substantially lower computational or hardware complexity and system latency.

\section{APPENDIX}

\section{A. Relationships Between Absolute-Relative-Error Bounds and Squared Amplitude-Response Error Bounds}

The squared amplitude-response error in (37) can also be expressed as

$$
\frac{N(\omega)}{D(\omega)}-F_{d}(\omega)=\omega^{2} e_{h}^{2}(\omega)+2 \omega \sqrt{F_{d}(\omega)} e_{h}(\omega)
$$

where $e_{h}(\omega)$ is the relative error which is given by

$$
e_{h}(\omega)=\frac{1}{|\omega|}\left[\sqrt{\frac{N(\omega)}{D(\omega)}}-\sqrt{F_{d}(\omega)}\right]
$$

For a differentiator, $F_{d}(\omega)=\omega^{2}$ and hence (55) becomes

$$
\frac{N(\omega)}{D(\omega)}-F_{d}(\omega)=\omega^{2}\left[e_{h}^{2}(\omega)+2 e_{h}(\omega)\right]
$$

Substituting (57) in (37) and simplifying, we get

$$
\begin{aligned}
& \left|e_{h}(\omega)+1\right| \leq \sqrt{1+\frac{\epsilon_{r}(\omega)}{\omega^{2}}} \\
& \left|e_{h}(\omega)+1\right| \geq \sqrt{1-\frac{\epsilon_{l}(\omega)}{\omega^{2}}}
\end{aligned}
$$

With the assumption that $\left|e_{h}(\omega)\right| \ll 1$, the term $\left[e_{h}(\omega)+1\right]$ is always positive. Consequently, (58) and (59) simplify to

$$
\begin{aligned}
& e_{h}(\omega) \leq \sqrt{1+\frac{\epsilon_{r}(\omega)}{\omega^{2}}}-1 \\
& e_{h}(\omega) \geq \sqrt{1-\frac{\epsilon_{l}(\omega)}{\omega^{2}}}-1
\end{aligned}
$$

If $\delta_{r}$ is the maximum relative error, we have

$$
\begin{aligned}
& e_{h}(\omega) \leq \delta_{r} \\
& e_{h}(\omega) \geq-\delta_{r}
\end{aligned}
$$

Equating (60) and (61) to (62) and (63), respectively, and simplifying, we get

$$
\begin{aligned}
\epsilon_{r}(\omega) & =\left(2 \delta_{r}+\delta_{r}^{2}\right) \omega^{2} \\
\epsilon_{l}(\omega) & =\left(2 \delta_{r}-\delta_{r}^{2}\right) \omega^{2}
\end{aligned}
$$

\section{REFERENCES}

[1] G. F. Franklin, J. D. Powell, and M. L. Workman, Digital Control of Dynamic Systems, 2nd ed. Reading, MA, USA: Addison-Wesley, 1990.

[2] P. Laguna, N. Thakor, P. Caminal, and R. Jane, "Low-pass differentiators for biological signals with known spectra: Application to ECG signal processing," IEEE Trans. Biomed. Eng., vol. 37, pp. 420-425, Apr. 1990.

[3] A. E. Marble, C. M. McIntyre, R. Hastings-James, and C. W. Hor, “A comparison of algorithms used in computing the derivative of the left ventricular pressure," IEEE Trans. Biomed. Eng., vol. BME-28, pp. 524-529, Jul. 1981.

[4] S. Shiro and A. Imidror, "Digital low-pass differentiation for biological signal processing," IEEE Trans. Biomed. Eng., vol. BME-29, pp. 686-693, Oct. 1982.

[5] M. I. Skolnik, Introduction to Radar Systems, 2nd ed. New York, NY, USA: McGraw-Hill, 1980.

[6] V. Torre and T. A. Poggio, "On edge detection," IEEE Trans. Pattern Anal. Mach. Intell., vol. PAMI-8, pp. 147-163, Mar. 1986.

[7] J. L. Bihan, "Novel class of digital integrators and differentiators," Electron. Lett., vol. 29, no. 11, pp. 971-973, May 1993.

[8] M. A. Al-Alaoui, "A class of second-order integrators and low-pass differentiators," IEEE Trans. Circuits Syst. I, Fundam. Theory Appl., vol. 42, no. 4, pp. 220-223, Apr. 1995.

[9] M. A. Al-Alaoui, "Linear phase low-pass IIR digital differentiators," IEEE Trans. Signal Process., vol. 55, no. 2, pp. 697-706, Feb. 2007.

[10] L. R. Rabiner and K. Steiglitz, "The design of wide-band recursive and nonrecursive digital differentiators," IEEE Trans. Audio Electroacoust., vol. AU-18, pp. 204-209, Jun. 1970.

[11] M. A. Al-Alaoui, "Novel digital integrator and differentiator," Electron. Lett., vol. 29, no. 4, pp. 376-378, Feb. 1993.

[12] N. Q. Ngo, "A new approach for the design of wideband digital integrator and differentiator," IEEE Trans. Circuits Syst. II, Exp. Briefs, vol. 53, no. 9, pp. 936-940, Sep. 2006.

[13] M. Gupta, M. Jain, and B. Kumar, "Novel class of stable wideband recursive digital integrators and differentiators," IET Signal Process., vol. 4 , no. 5 , pp. 560-566, Oct. 2010. 
[14] D. K. Upadhyay and R. K. Singh, "Recursive wideband digital differentiator and integrator," Electron. Lett., vol. 47, no. 11, pp. 647-648, May 2011.

[15] M. Jain, M. Gupta, and N. Jain, "Linear phase second order recursive digital integrators and differentiators," Radioengineering, vol. 21, no. 2, Jun. 2012

[16] X. P. Lai, Z. Lin, and H. K. Kwan, "A sequential minimization procedure for minimax design of IIR filters based on second-order factor updates," IEEE Trans. Circuits Syst. II, vol. 58, pp. 51-55, Jan. 2011.

[17] C.-C. Tseng, "Stable IIR diferentiator design using iterative quadratic programming approach," Signal Process., vol. 80, no. 5, pp. 857-866, 2000.

[18] D. K. Upadhyay, "Recursive wideband digital differentiators," Electron. Lett., vol. 46, no. 25, pp. 1661-1662, 2010.

[19] D. K. Upadhyay, "Class of recursive wideband digital differentiators and integrators," Radioengineering, vol. 21, no. 3, Sep. 2012.

[20] R. C. Nongpiur, D. J. Shpak, and A. Antoniou, "Improved design method for nearly linear-phase IIR filters using constrained optimization," IEEE Trans. Signal Process., vol. 61, no. 4, pp. 895-906, Feb. 2013.

[21] W.-S. Lu and T. Hinamoto, "Optimal design of IIR digital filters with robust stability using conic-quadratic-programming updates," IEEE Trans. Signal Process., vol. 51, no. 6, pp. 1581-1592, Jun. 2003.

[22] A. Antoniou, Digital Signal Processing: Signals, Systems, and Filters. New York, NY, USA: McGraw-Hill, 2005.

[23] A. Antoniou and W.-S. Lu, Practical Optimization: Algorithms and Engineering Applications. New York, NY, USA: Springer, 2007

[24] J. F. Sturm, "Using SeDuMi1.02, a MATLAB toolbox for optimization over symmetric cones," Optimization Methods Softw., vol. 11-12, pp. $625-653,1999$

[25] L. Rabiner, N. Graham, and H. Helms, "Linear programming design of IIR digital filters with arbitrary magnitude function," IEEE Trans. Acoust., Speech, Signal Process., vol. ASSP-22, no. 2, pp. 117-123, Feb. 1974.

[26] A. H. Sayed and T. Kailath, "A survey of spectral factorization methods," Numerical Linear Algebra Appl., vol. 8, pp. 467-496, 2001.

[27] R. C. Nongpiur, D. J. Shpak, and A. Antoniou, "Poles and zeros for examples and additional examples and results for digital differentiators," [Online]. Available: http://www.ece.uvic.ca/ andreas/ JournalPapers/Poles_and_Zeros_and_Additional_Results_for_Digital Differentiators.pdf

[28] I. Selesnick, "Maximally flat lowpass digital differentiators," IEEE Trans. Circuits Syst. II, vol. 49, no. 3, pp. 219-223, Mar. 2002

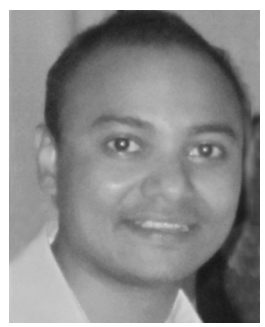

Rajeev C. Nongpiur (S'01-AM'05-M'12) received the B.Tech. degree in electronics and communications engineering from the Indian Institute of Technology, Kharagpur, India, in 1998 and the $\mathrm{Ph} . \mathrm{D}$. degree from the University of Victoria, British Columbia, Canada, in 2005. From 1998 to 2000, he worked as a Systems Engineer at Wipro Technologies, from 2004 to 2008 as a Research Scientist at QNX Software Systems, and from 2008 to 2010 as Senior DSP Engineer with Unication Co., Ltd., Vancouver, Canada. He is currently serving as a Research Associate in the Department of Electrical and Computer Engineering, University of Victoria, British Columbia, Canada. His research interests are in the areas of signal processing for digital communications, multimedia, and biomedical applications. He is the author of more than 15 patents in the area of audio signal processing.

Dr. Nongpiur is a member of the IEEE Circuits and Systems and Signal Processing Societies.

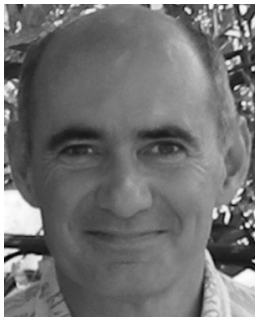

Dale J. Shpak (S'79-M'86-SM'09) received the B.Sc. (Elec. Eng.) from the University of Calgary, Canada, in 1980. From 1980 to 1982 , he worked as an engineer for the City of Calgary Electric System while earning his M.Eng. in electronics. Between 1982 and 1987, he performed research on computer systems, microelectronics, and DSP algorithms and implementation. From 1987 to 1989 , he received his Ph.D. from the University of Victoria, Canada. Starting in 1988, he served as a Professor with the Department of Engineering, Royal Roads Military College and returned to industry when it closed in 1995.

Since 1989 he has held a faculty position at the University of Victoria in addition to his other professional activities. As an Adjunct Professor of Electrical and Computer Engineering, he receives ongoing NSERC funding for research programs with his graduate students.

He joined the Department of Computer Science at Camosun College in 1999. He has instructed over thirty different courses including object-oriented programming, computer networks, digital circuit design, digital filters, materials science, software engineering, and real-time and concurrent systems.

$\mathrm{He}$ held several positions in industry where he developed algorithms, software, circuits, networking systems, and embedded systems. Since 1984, he has served as a consultant and develops software and embedded systems for products including audio processing, wireless sensing and control, and remote sensing. He is a Principal Developer of award-winning products, including the Filter Design Toolbox for MATLAB ${ }^{\mathrm{TM}}$.

Dr. Shpak is a Senior Member of the IEEE and a Member of the Association of Professional Engineers of the Province of British Columbia. His principal research interests are in the areas of signal processing for communications and audio, design and implementation of embedded systems, and digital filter design.

He also develops free software for music education.

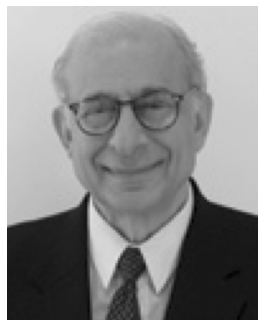

Andreas Antoniou (M'69-SM'79-F'82-LF'04) received the B.Sc.(Eng.) and Ph.D. degrees in electrical engineering from the University of London in 1963 and 1966, respectively, and is a Fellow of the IET and IEEE. He taught at Concordia University from 1970 to 1983 , was the founding Chair of the Department of Electrical and Computer Engineering, University of Victoria, B.C., Canada, from 1983 to 1990, and is now Professor Emeritus. His teaching and research interests are in the area of digital signal processing. He is the author of Digital Signal Processing: Signals, Systems, and Filters (McGraw-Hill, 2005) and the co-author, with Wu-Sheng Lu, of Practical Optimization: Algorithms and Engineering Applications (Springer, 2007).

Dr. Antoniou served first as Associate Editor and after that as Chief Editor for the IEEE TRANSACTIONS ON CIRCUITS AND SYSTEMS from 1983 to 1987, as a Distinguished Lecturer of the IEEE Signal Processing and the Circuits and Systems Societies during 2003-2004 and 2006-2007, respectively, and as General Chair of the 2004 International Symposium on Circuits and Systems.

He was awarded the CAS Golden Jubilee Medal by the IEEE Circuits and Systems Society, the B.C. Science Council Chairman's Award for Career Achievement for 2000, the Doctor Honoris Causa Degree by the National Technical University, Athens, Greece, in 2002, the IEEE Circuits and Systems Society Technical Achievement Award for 2005, the 2008 IEEE Canada Outstanding Engineering Educator Silver Medal, the IEEE Circuits and Systems Society Education Award for 2009, and the 2011 Craigdarroch Gold Medal for Career Achievement and the 2011 Legacy Award for Research, both from the University of Victoria, Victoria, Canada. 